\title{
Glucoherb versus metformin on glycemic markers and glycosylated hemoglobin in prediabetes patients; a clinical trial study
}

\author{
Iman Izadi $^{\circledR}$, Rahil Riahi Samani* ${ }^{\circledR}$, Afsaneh Malekpour Tehrani ${ }^{\circledR}$, Marziyeh Dehghani ${ }^{\circledR}$, \\ Alireza Jafari ${ }^{\mathbb{D}}$ \\ Department of Internal Medicine, School of Medicine, Shahrekord University of Medical Sciences, Shahrekord, Iran
}

\section{A R T I C L E I N F O}

Article Type:

Original

\section{Article History:}

Received: 19 March 2020

Accepted: 3 July 2020

Published online: 24 July 2020

Keywords:

Glucoherb, Diabetes mellitus, Fasting blood sugar, Glycated hemoglobin, Metformin, Pre-diabetic

\begin{abstract}
A B S T R A C T
Introduction: There is a high risk of diabetes mellitus in pre-diabetic patients.

Objectives: The aim of the study was to compare the effect of the extracts of these herbs in the formulation of Glucoherb supplementation versus metformin in pre-diabetes patients on glycemic markers and glycosylated hemoglobin.

Patients and Methods: Pre-diabetic patients who had indication of drug treatment according to the criteria of the American Diabetes Association, were selected and randomly divided into two groups of Glucoherb and metformin. Body mass index (BMI), fasting blood sugar (FBS), 2 hours postprandial blood glucose (BS2PP) and glycosylated hemoglobin (HbA1c) were measured before and after the intervention.

Results: The mean BMI, FBS, BS2PP and HbAlc levels were significantly decreased after intervention in both Glucoherb and metformin groups $(P<0.05)$ without any significant difference between them $(P<0.05)$.

Conclusion: Glucoherb showed similar efficacy to metformin in reducing blood glucose, BMI and HbAlc in pre-diabetic patients.

Trial registration: The trial protocol was approved in the Iranian Registry of Clinical Trials (identifier: IRCT20190125042486N2; https://en.irct.ir/trial/37877, ethical code; IR.SKUMS. REC.1397.266).
\end{abstract}

\section{Implication for health policy/practice/research/medical education:}

In a clinical trial, the effect of Glucoherb supplementation as a combination of some herbal drugs versus metformin on glycemic markers and glycosylated hemoglobin in pre-diabetes patients was compared. We found, the body mass index (BMI), fasting blood sugar (FBS), 2 hours postprandial blood glucose (BS2PP) and glycosylated hemoglobin (HbAlc) levels significantly decreased after intervention in both Glucoherb and metformin groups without any significant difference between them. We concluded that Glucoherb had similar efficacy to metformin in reducing blood glucose, BMI and HblAC in pre-diabetic patients. Please cite this paper as: Izadi I, Riahi Samani R, Malekpour Tehrani A, Dehghani M, Jafari A. Glucoherb versus metformin on glycemic markers and glycosylated hemoglobin in prediabetes patients; a clinical trial study. J Nephropharmacol. 2021;10(1):08. DOI: $10.34172 /$ npj.2021.08.

\section{Introduction}

Diabetes mellitus is the most common metabolic disease around the world. It is known as the largest epidemic of the century among all chronic diseases. There are currently around 415 million people with diabetes mellitus worldwide and it is estimated to reach 642 million by 2040 (1). There are also considering unidentified pre-diabetes patients, which seems to be much higher than diabetics (prevalence rate of more than 16\%) (2).

In diabetes mellitus, hyperglycemia for longtime causes microvascular complications of diabetes which can affect various organs of the body such as the kidneys and eyes (3). It is worthy to state that diabetes mellitus is the most important cause of blindness in people aged 25-75 years and also the most common cause of amputation in the United States. Moreover, 35\% of patients with chronic renal failure and dialysis are diabetic (4).

Diabetes mellitus is classified into two main forms of type 1 and type 2 . In type 1 diabetes mellitus, which accounts for about $5 \%$ to $10 \%$ of cases, the disease is caused by a disorder in the immune system of insulin-producing cells in the pancreas. Type 2 diabetes mellitus, which is 
accounting for approximately 90 to 95 percent of diabetes cases, is caused by the inability of muscle cells to respond to insulin (insulin resistance) (5). American Diabetes Association (ADA) stated that it is necessary to have at least one of the following three cases to be considered as pre-diabetes patients;

1. Plasma glucose in the fasting state $\geq 100-125 \mathrm{mg} / \mathrm{dL}$

2. Two hours glucose in oral glucose tolerance testing $\geq$ 140-199 mg/dL

3. HbA1c between $5.7-6.4 \%$ (6).

According to the ADA, people with high blood pressure should lose $7 \%$ of their original weight and increase their physical exercise to reach 150 minutes/ weekly. Among these individuals, patients with special conditions are treated with medication (5). Studies have shown that taking herbal medicines can reduce blood sugar and prevent the side effects of taking anti-diabetic drugs. Today the use of herbal medicine as adjuvant therapy for diabetes is common since around 26 plant species used in the treatment of diabetes, walnut (Juglans regia), salt grass (Atriplex halimus), olive (Olea europaea) and nettle (Urtica dioica) are strongly recommended. Studies have shown the anti-diabetic effects of olive leaf extract $(7,8)$, walnut leaf extract $(9,10)$, salt grass $(11)$ and nettle (12). In the formulation of Glucoherb drug, the walnut leaves, olive leaves, salt grass and nettle have been used. In a previous study by Said et al, the safety and anti-diabetic effects of Glucoherb were shown in 16 volunteers with a recent diagnosis of type 2 diabetes (13).

\section{Objectives}

The present study was designed to investigate the efficacy of this drug in pre-diabetic patients.

\section{Patients and Methods}

Study design

In this clinical trial study the pre-diabetic individuals who needed medical treatment according to the ADA, were selected by easy sampling method from the diabetes center of Shahrekord in 2019.

Inclusion criteria include non-smoking, nonuse of alcohol, non-use of anti-diabetic and insulin drugs, absence of pregnancy or lactation, non-use of multivitamin supplements, antioxidants or omega-3 in the last three months, lack of kidney, liver and thyroid and parathyroid, gastrointestinal and cardiac diseases, lack of cancer and lack of taking medications that affect blood sugar, such as steroids and also presence of prediabetic condition (glycosylated hemoglobin between $5.7 \%-6.4 \%$; fasting blood sugar of $100-125 \mathrm{mg} / \mathrm{dL}$ and 2 hours postprandial blood glucose (BS2PP $\geq 140-199$ $\mathrm{mg} / \mathrm{dL})$. The indications for treatment of pre-diabetes according to the ADA include body mass index (BMI) more than $35 \mathrm{k} / \mathrm{m}^{2}$, fasting blood sugar more than 110 $\mathrm{mg} / \mathrm{dL}$, a history of gestational diabetes and age of under 60 years. Exclusion criteria included allergic reaction to the supplement, withdrawal of the patient to participate in the study, acceptance of less than $80 \%$ of the intervention (consumption of less than $80 \%$ of the total supplement that should be taken during three months of intervention was considered as low admission) and change in the type of daily used medication.

Initially, written consent was obtained from patients. BMI was calculated and then blood sampling obtained in fasting state and FBS, BS2PP and HbA1c levels. In this study, blinding was not performed and patients were introduced to a nurse in the diabetes unit, where they were randomized to receive metformin or Glucoherb. The dose of medication was based on the physician's opinion. The acceptance rate of supplementation by patients was assessed by telephone calls as well as by returning empty supplement boxes, since taking more than $80 \%$ of the supplement at the end of 12 weeks was considered by the patient as the acceptance of drug. After 3 months, blood samples were taken to re-test the mentioned parameters.

\section{Ethical issues}

The research conducted in accordance with the tenets of the Declaration of Helsinki. The institutional ethical committee at Shahrekord University of Medical Sciences accepted all study protocols (IR.SKUMS.REC.1397.266). Accordingly, written informed consent was taken from all participants before any intervention. This study was part of the internal medicine residential thesis of Iman Izadi at this university. The trial protocol was also approved by the Iranian Registry of Clinical Trial (identifier: IRCT20190125042486N2; https://en.irct.ir/trial/37877).

\section{Statistical analysis}

Data analyses were carried out using SPSS software; descriptive statistics (including mean, standard deviation, frequency and percentage) and inferential statistics (including independent sample $t$ test, chi-square test, Mann-Whitney $U$ test and Wilcoxon signed-rank test) were applied for data analysis. $P<0.05$ was considered as significant difference.

Results

In the present clinical trial, 72 pre-diabetic patients referred to the diabetic center of Shahrekord, who had the indications for drug treatment according to the criteria of the ADA, were randomly assigned to two groups of Glucoherb $(n=36)$ and metformin $(n=36)$ (Figure 1$)$. The metformin group consisted of three males $(8.3 \%)$ and 33 females $(91.7 \%)$ and the Glucoherb group included four males (11.1\%) and 32 females (88.9\%). The result of chi-square test did not show any significant difference in gender distribution between Glucoherb and metformin groups $(P>0.05$; Table 1$)$.

The mean age of all patients was $47.43 \pm 6.75$ years. The mean age of patients in the metformin group was $48.06 \pm$ 6.19 years and it was $46.81 \pm 7.30$ years in the Glucoherb 


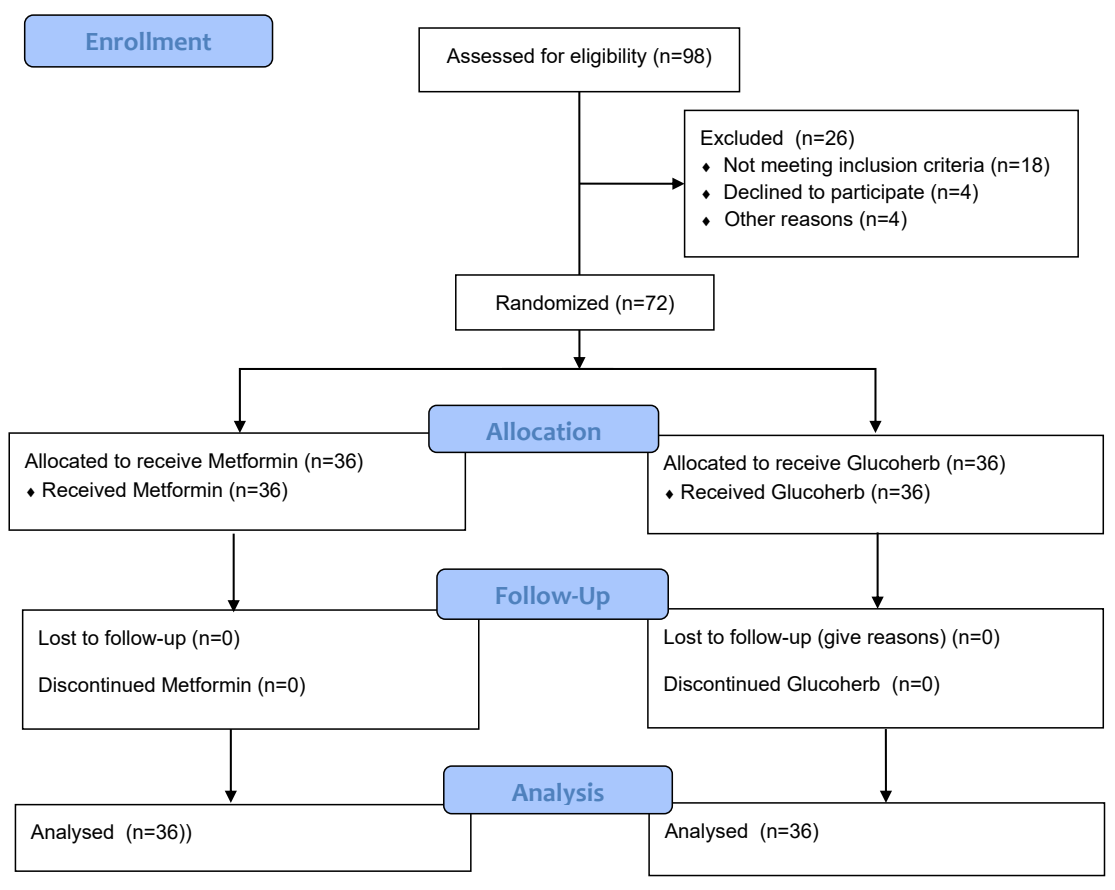

Figure 1. CONSORT flow chart showing the flow of patients through the trial.

group, which did not show a significant difference $(P>0.05$; Table 2).

The mean \pm SD of BMI, FBS, BS2PP and HbAlc in the studied groups are shown in Table 3. The mean levels of $\mathrm{BMI}, \mathrm{FBS}, \mathrm{BS} 2 \mathrm{PP}$ and $\mathrm{HbAlc}$ did not show any significant difference before the intervention between Glucoherb and metformin groups $(P>0.05)$. The mean BMI, FBS, BS2PP and HbA1c levels significantly decreased after intervention (in comparison with before the intervention) in both Glucoherb and metformin groups $(P<0.05)$. After the intervention, the mean levels of BMI, FBS, BS2PP and HbAlc did not show any significant difference between Glucoherb and metformin groups $(P>0.05)$.

\section{Discussion}

In the present clinical trial, the effect of Glucoherb supplementation and metformin on glycemic markers

Table 1. Gender distribution of patients in studied groups

\begin{tabular}{lcccc}
\hline Group & Male & Female & Total & P value \\
\hline Metformin & $3(8.3 \%)$ & $33(91.7 \%)$ & $36(100 \%)$ & \\
Glucoherb & $4(11.1 \%)$ & $32(88.8 \%)$ & $36(100 \%)$ & 0.500 \\
\hline
\end{tabular}

Table 2. Mean age of patients between different studied groups

\begin{tabular}{lccc}
\hline Group & Mean & Standard deviation & $P$ value \\
\hline Metformin & 48.06 & 6.19 & \\
Glucoherb & 46.81 & 7.30 & 0.431 \\
Total & 47.43 & 7.75 & \\
\hline
\end{tabular}

and glycosylated hemoglobin in pre-diabetes patients was compared. Seventy-two pre-diabetic patients who had the indications for drug treatment according to the criteria of the ADA, were randomly assigned into two groups of Glucoherb and metformin. The two groups were homogeneous and had no significant difference in terms of mean age and gender distribution. According to our results, the treatment of pre-diabetic patients with metformin for three months caused a significant decrease in mean BMI, FBS, BS2PP and HbA1c compared to those levels before the intervention. In line with our results, several clinical trials have also shown the lowering effect of metformin on blood sugar, HbA1c, and BMI (14-16).

Metformin is currently one of the best drugs recommended for delayed or prevented type 2 diabetes in high-risk individuals. Regardless of its gastrointestinal side effects, the drug is well tolerated and low in cost (7). Preventing or restoring progressive insulin resistance and dysfunction of $\beta$-cells associated with dysglycemia is the main key for delaying or preventing the conversion of pre-diabetes into type 2 clinical diabetes (17). Metformin primarily acts by increasing the activity of insulin in the liver to reduce the production of hepatic glucose (18). Improvement of insulin activity in skeletal muscle also plays a role in the therapeutic effects of metformin, which mainly leads to non-oxidative consumption of glucose (glycogen formation) (19). These functions together can result in lower blood sugar, with very little potential for induction of hypoglycemia (17). Increased anaerobic metabolism in the intestinal wall is also probably one of the important anti-hyperglycemic mechanisms of metformin 
Table 3. Mean BMI $\left(\mathrm{kg} / \mathrm{m}^{2}\right)$, FBS $(\mathrm{mg} / \mathrm{dL})$, BS2PP $(\mathrm{mg} / \mathrm{dL})$ and $\mathrm{HbA} 1 \mathrm{c}(\%)$ in different studied groups

\begin{tabular}{|c|c|c|c|c|}
\hline Variable & & Metformin group & Glucoherb group & $P$ value \\
\hline \multirow{3}{*}{ BMI } & Before intervention & $29.47 \pm 4.70$ & $29.50 \pm 5.00$ & 0.892 \\
\hline & After intervention & $28.77 \pm 4.83$ & $28.68 \pm 4.66$ & 0.942 \\
\hline & $P$ value & 0.005 & 0.002 & - \\
\hline \multirow{3}{*}{ FBS } & Before intervention & $114.72 \pm 5.75$ & $112.89 \pm 4.52$ & 0.055 \\
\hline & After intervention & $105.67 \pm 6.44$ & $103.69 \pm 10.45$ & 0.269 \\
\hline & $P$ value & $<0.001$ & $<0.001$ & - \\
\hline \multirow{3}{*}{ BS2PP } & Before intervention & $133.22 \pm 23.74$ & $125.92 \pm 19.02$ & 0.125 \\
\hline & After intervention & $119.69 \pm 21.93$ & $119.78 \pm 26.93$ & 0.857 \\
\hline & $P$ value & 0.001 & 0.001 & - \\
\hline \multirow{3}{*}{$\mathrm{HbA} 1 \mathrm{c}$} & Before intervention & $6.00 \pm 0.62$ & $5.92 \pm 0.47$ & 0.667 \\
\hline & After intervention & $5.64 \pm 0.49$ & $5.71 \pm 0.47$ & 0.879 \\
\hline & $P$ value & $<0.001$ & $<0.002$ & - \\
\hline
\end{tabular}

(20). In addition, metformin has been shown to increase the level of circulating glucagon-1 like peptide (GLP-1) in the blood by directly increasing GLP-1 secretion or reducing the activity of dipeptidyl peptidase-4 (DPP4). DPP4 is mainly responsible for inactivating GLP-1 in tissues and blood circulation (17). Metformin may also reregulate the expression of GLP-1 receptors on the surface of pancreatic $\beta$ cells (21). GLP-1 is of incretin hormones that is secreted from the intestines after digestion and increases insulin secretion and regulates blood sugar. GLP-1 is also able to inhibit cell death and increase the proliferation of pancreatic beta cells (17). From a cellular point of view, metformin appears to reduce the production of glucose in the liver by inhibiting the complex of cellular respiration chain number 1 in the mitochondria of liver cells, which causes a temporary decrease in cellular energy in the liver, which in turn increases AMP activated protein kinase (AMPK). AMPK is itself a sensitive receptor for metabolism and energy in liver cells, since the elevated AMPK inhibits the transcription of gluconeogenesis cycles and thus inhibits glucose production in liver cells (22).

Studies have shown that metformin has a multifactorial effect on appetite and weight and includes changes in the physiology of the hypothalamus, including leptin and insulin sensitivity. In addition, new studies on obesity have confirmed the physiological alteration of the gastrointestinal tract and the rhythm of day and night by metformin, which not only affects food intake, but also regulates fat oxidation and fat storage in the liver, skeletal muscle and fat mass (23).

According to our results, the treatment of prediabetic patients with Glucoherb for 3 months caused a significant decrease in mean BMI, FBS, BS2PP and $\mathrm{HbA1c}$ compared to the levels before the intervention. In a study conducted by Said et al, the anti-diabetic effect of Glucoherb was observed in cell culture media and rats and also in a clinical trial. In the cell culture medium, Glucoherb reduced glucose uptake by yeast cells. In rats,
Glucoherb also inhibited the intestinal absorption of glucose by $49 \%$. Besides, in the animal model of diabetes caused by streptozotocin, the treatment with Glucoherb for 2-3 weeks significantly reduced blood sugar levels. Additionally, in 16 volunteers aged 48-67 years, with the recent diagnosis of type 2 diabetes who were only treated with diet, taking three Glucoherb tablets per day for 4 weeks caused a significant reduction in blood sugar and HbAlc (13). One of the strengths of the present study compared to the above study was larger samples (36 people compared to 16 people) and also comparison of Glucoherb with metformin (standard drug for pre-diabetic patients). We observed that Glucoherb had similar efficacy to metformin in reducing FBS, BS2PP, HbA1c and BMI.

As mentioned earlier, in the formulation of Glucoherb, walnut leaves, olive leaves, salt grass and nettle were used which their anti-diabetic effects have been shown in animal studies as well as clinical trials.

Regarding walnut leaf extract, in a study by Al-Attar and Alsalmi, the treatment of diabetic rats with walnut leaf extract significantly reduced blood sugar and blood lipids. Olive leaf extract has also reduced lipid peroxidation and boosted the activity of antioxidant enzymes in diabetic mice. They found, olive leaf extract protects pancreatic beta cells by inhibiting oxidative stress (7). In a study by Arab et al, walnut consumers showed lower fasting blood glucose and HbAlc compared with non-nut consumers. (24). Dzhafavora et al observed that the treatment of diabetic rats with walnut leaf extract significantly reduced blood sugar and $\mathrm{HbAlc}$ and improved pancreatic tissue damage (9). In another study, the treatment of streptozotocin induced diabetic rats with walnut leaf extract significantly reduced sugar, cholesterol, lowdensity lipoprotein (LDL-C) and serum triglycerides and significantly increased high-density lipoprotein (HDL-C) (10). A previous study also showed that walnut leaf extract reduced FBS, $\mathrm{HbA1c}$, total cholesterol and triglycerides in type 2 diabetic patients. In this study, walnut leaf extract showed no liver and kidney side effects except for some 
gastrointestinal side effects (especially mild diarrhea) (25).

Regarding the olive leaf extract, Abd El-Rahman et al reported that the treatment of diabetic rats with olive leaf extract significantly reduced glucose, cholesterol and triglycerides and significantly increased HDL-C (8). Likewise, the study by de Bock et al, on overweight middle-aged men, showed treatment with olive leaf extract improved insulin sensitivity by 15 percent and improved beta cell response by 28 percent compared with the control group. In this study, the activity of olive leaf extract was related to the increased levels of protein 1 binding insulinlike growth factor (IGFBP-1) and protein 2 binding insulin-like growth factor (IGFBP-2) (26). Of six IGFBPs, the IGFBP-1 and IGFBP-2 are the most important IGFBPs which play an important role in insulin and glucose metabolism. IGFBP-1 and IGFBP-2 are mainly produced in the liver and bind to insulin like growth factors I and II (IGF-I and IGF-II). There are considerable evidences that impaired IGFBP-1 and IGFBP-2 regulation may play a role in metabolic development, including diabetes (5).

In relation to salt grass, Chikhi et al observed that the treatment of streptozotocin-induced diabetes in rats which was treated with salt grass extract, showed a significant blood and liver glucose reduction (11). Regarding Urtica dioica extract, the study by Dar et al showed Urtica dioica leaves extract reduced blood glucose levels during GTT in Wistar rats significantly $(P<0.001)(27)$.

According to the present study, Glucoherbs showed similar effects to metformin in lowering blood sugar, $\mathrm{HbA1c}$ and BMI in pre-diabetic patients and can therefore be used as an alternative or adjuvant therapy to improve response and prevent type 2 diabetes in pre-diabetics. However, as proved in the previous studies, herb-drug interactions may be a double-edged sword faced the patients with both adverse drug effects and also desirable positive effects (28). Hence, further studies are necessary to better understand the interaction between different groups of drugs and herbs to avoid adverse side effect of the simultaneous use of drug and herb.

\section{Conclusion}

According to the results of our study, the treatment of pre-diabetics with Glucoherb and metformin for three months resulted in a significant decrease in FBS, BS2PP, $\mathrm{HbAlc}$ and BMI. In addition, metformin and Glucoherb showed similar efficacy, therefore the FBS, BS2PP, HbA1c, and BMI values did not differ significantly after the intervention between the two groups.

\section{Limitations of the study}

Low drug compliance and adherence to treatment in patients that lead to exclusion from the study.

\section{Authors' contribution}

RRS, II, AMT were the principal investigators of the study and they were included in preparing the concept and designing of the study. RRS, II, MD, AJ were involved in performing study tests and data acquiring. SM and AMT revised the manuscript. All authors participated in preparing the final draft of the manuscript.

\section{Conflicts of interest}

The authors declare that no conflict of interest.

\section{Ethical considerations}

The research was extracted from the residential thesis of Iman Izadi at Shahrekord University of Medical Sciences. Ethical issues (double publication, data fabrication and plagiarism) have been completely observed by the authors.

\section{Funding/Support}

The study was part of MD thesis of Iman Izadi at Shahrekord University of Medical Sciences supported by the university.

\section{References}

1. Baynes H. Classification, pathophysiology, diagnosis and management of diabetes mellitus. J Diabetes Metab. 2015; 6:1-9. doi:10.4172/2155-6156.1000541.

2. Tripathi BK, Srivastava AK. Diabetes mellitus: complications and therapeutics. Med Sci Monit. 2006;12:RA130-47.

3. ADA. Type 2 diabetes in children and adolescents. Pediatrics. 2000;105:671-680.

4. CDC. National diabetes fact sheet, United States, 2005. http://www.cdc.gov/diabetes/pubs/pdf/ndfs_2005.pdf Accessed 20 September 2006).

5. American Diabetes Association. Diagnosis and classification of diabetes mellitus. Diabetes Care. 2013;36:67-74.

6. Tang O, Matsushita K, Coresh J, Sharrett AR, McEvoy JW, Windham BG, et al. Mortality implications of prediabetes and diabetes in older adults. Diabetes Care. 2020;43(2):382388. doi:10.2337/dc19-1221

7. Al-Attar AM, Alsalmi FA. Effect of Olea europaea leaves extract on streptozotocin induced diabetes in male albino rats. Saudi J Biol Sci. 2019;26:118-128. doi: 10.1016/j. sjbs.2017.03.002.

8. Abd El-Rahman HSM. The effect of olive leaf extract and A-Tocopherol on nephroprotective activity in rats. J Nutr Food Sci. 2016;6:479.

9. Dzhafarova RE, Garaev GSh, Dzhafarkulieva ZS. Georgian Med News. 2009;(170):110-114.

10. Mollica A, Zengin G, Locatelli M, Stefanucci A, Macedonio $\mathrm{G}$, Bellagamba $\mathrm{G}$, et al. An assessment of the nutraceutical potential of Juglans regia L. leaf powder in diabetic rats. Food Chem Toxicol. 2017;107:554-64. doi: 10.1016/j. fct.2017.03.056

11. Chikhi I, Allali H, Tabti B. Antidiabetic activity of aqueous leaf extract of Atriplex halimus L.(Chenopodiaceae) in streptozotocin-induced diabetic rats. Asian Pacific J Trop Dis. 2014;4:181-4. doi:10.1016/S2222-1808(14)60501-6.

12. El Haouari M, Rosado J. Phytochemical anti-diabetic and cardiovascular properties of Urtica dioica L. (Urticaceae): A Review. Mini Rev Med Chem 2019;19:63-71. doi: 10.2174/1 389557518666180924121528. 
13. Said O, Fulder S, Khalil K, Azaizeh H, Kassis E, Saad B. Maintaining a physiological blood glucose level with 'glucolevel', a combination of four anti-diabetes plants used in the traditional Arab herbal medicine. Evid Based Complem Altern Med. 2008;5:421-428. doi:10.1093/ecam/ nem 047.

14. Solymar M, Ivic I, Poto L, Hegyi P, Garami A, Hartman $\mathrm{P}$, et al. Metformin induces significant reduction of body weight, total cholesterol and LDL levels in the elderly-A meta-analysis. PLoS One. 2018;13:e0207947. doi: 10.1371/ journal.pone.0207947.

15. Khan A, McLoughney C, Ahmed A. The effect of metformin on blood glucose control in overweight patients with type 1 diabetes. Diabetic Med. 2006;23:1079-84. doi: 10.1111/j.1464-5491.2006.01966.x.

16. Fonseca VA, Theuma P, Mudaliar S, Leissinger CA, Clejan S, Henry RR. Diabetes treatments have differential effects on nontraditional cardiovascular risk factors. J Diabetes Complications. 2006;20:14-20. doi: 10.1016/j. jdiacomp.2005.05.009.

17. Hostalek U, Gwilt M, Hildemann S. Therapeutic use of metformin in prediabetes and diabetes prevention. Drugs. 2015;75:1071-1094. doi: 10.1007\%2Fs40265-015-0416-8.

18. Natali A, Ferrannini E. Effects of metformin and thiazolidinediones on suppression of hepatic glucose production and stimulation of glucose uptake in type 2 diabetes: a systematic review. Diabetologia. 2006;49:434441. doi: 10.1007/s00125-006-0141-7.

19. Johnson A, Webster J, Sum C, Heseltine C, Argyraki M, Cooper B, et al. The impact of metformin therapy on hepatic glucose production and skeletal muscle glycogen synthase activity in overweight type II diabetic patients. Metabolism. 1993;42:1217-22. doi: 10.1016/0026-0495(93)90284-u.

20. Bailey C, Mynett K, Page T. Importance of the intestine as a site of metformin-stimulated glucose utilization. $\mathrm{Br}$ J Pharmacol. 1994;112:671-5. doi: 10.1111\%2Fj.14765381.1994.tb13128.x.
21. Thondam S, Cross A, Cuthbertson DJ, Wilding JP, Dausi C. Effects of chronic treatment with metformin on dipeptidyl peptidase- 4 activity, glucagon-like peptide 1 and ghrelin in obese patients with type 2 diabetes mellitus. Diabetic Med 2012;29:205-10 doi:10.1111/j.1464-5491.2012.03675.x.

22. Viollet B, Guigas B, Garcia N, Lecrec J, Foretz M, Andreeli F. Cellular and molecular mechanisms of metformin: an overview. Clin Sci. 2011;122:253-270. doi: 10.1042/ cs20110386.

23. Malin SK, Kashyap DR. Effects of metformin on weight loss: potential mechanisms. Curr Opin Endocrinol Diabetes Obesity. 2014;21:323-9.

24. Arab L, Dhaliwal S, Carley J, Larios A, Jackson N, Elashoff D. Association between Walnut consumption and diabetes risk in NHANES. Diabetes Metab Res Rev. 2018;34:e3031. FF. doi: 10.1002\%2Fdmrr.3031.

25. Hosseini, S, Jamshidi L, Mehrzadi S, Mohammad K, Najmizadeh R, Alimoradi H, et al. Effects of Juglans regia L. leaf extract on hyperglycemia and lipid profiles in type two diabetic patients: a randomized double-blind, placebo-controlled clinical trial. J Ethnopharmacol. 2014;152(3):451-456. doi: 10.1016/j.jep.2014.01.012.

26. de Bock M, Derraik J, Brennan C, Biggs J, Morgan P, Hodgkinson S, et al. Olive (Olea europaea L.) leaf polyphenols improve insulin sensitivity in middle-aged overweight men: a randomized, placebo-controlled, crossover trial. PloS One. 2013;8:e57622. doi: 10.1371/ journal.pone.0057622.

27. Dar SA, Ganai F, Yousuf A, Balkhi M, Bhat T, Sharma P. Pharmacological and toxicological evaluation of Urtica dioica. Pharm Biol. 2013;51:170-80. doi: 10.3109/13880209.2012.715172.

28. Gupta RC, Chang D, Nammi S, Bensoussan A, Bilinski K, Roufogalis BD. Interactions between antidiabetic drugs and herbs: an overview of mechanisms of action and clinical implications. Diabetol Metab Syndr. 2017;9:59. doi: 10.1186/s13098-017-0254-9.

Copyright $\odot 2021$ The Author(s); Published by Published by Society of Diabetic Nephropathy Prevention. This is an open-access article distributed under the terms of the Creative Commons Attribution License (http://creativecommons.org/licenses/by/4.0), which permits unrestricted use, distribution, and reproduction in any medium, provided the original work is properly cited. 